\title{
Remote Health Monitoring System using Cloud and Mobile Andriod Application
}

\author{
Tej Raj ${ }^{1}$, Mukesh Pathela ${ }^{2}$, and Champa Devi ${ }^{3}$ \\ ${ }^{1,2}$ DBIT Dehradun, ${ }^{3}$ BFIT Dehradun, India
}

\section{ABSTRACT}

Healthcare or remote health monitoring system is getting popular due to the present scenario like COVID-19. It is difficult for the regular checkup of patients to visit in the health care unit as well as for doctors to diagnose the patient from time to time. In such cases, there must be a solution that will continuously monitor the patient's health, store the history of a patient, and easily assessable to the health care unit or doctor at remote places and this can be possible by the use of wireless technology. Instead of monitor the patient's health inside the hospital rooms, with the help of sensors, intelligent edge devices, and cloud computing it is possible to monitor the patient's health while doing its normal activities at home. In this paper, we have presented the system in which, with the help of sensor technology and edge device raspberry pi to monitor the patient's illness and then ingest the sensor data from raspberry pi to cloud platform for further processing of that data. We have used the electrocardiogram, heart rate, and temperature sensors for measuring the vital illness parameter of the body. This system is helpful for people with chronological illness, victims of the accident, elder people who need continuous monitoring of their health.

\section{KEY WORDS: IOT(INTERNET OF THINGS), REMOTE HEALTH MONITORING, E-HEALTH, ECG, MCP3008, PULSE SENSOR.}

\section{INTRODUCTION}

Population blast specifically in a developing country has brought numerous challenges in health care systems also in a present scenario like COVID-19 it leads to scarcity of medical resources and paralyzed our medical system. This makes it discretionary for us to address these challenges and equip the medical system with the latest technology for load balancing for doctors as well as other medical staff. With the evolution of wireless technology and connectivity with wearable sensors on the patient's body, it is easy to monitor the patient's health from a remote place. This also reduces the burden on medical resources and also feasible for the doctors to take care of the patient. This will reduce manual continuous monitoring of the patient, also with the help of the critical checkpoints of patients, an alert system can be made and with the help of this, some kind of message can

Biosc Biotech Res Comm P-ISSN: 0974-6455 E-ISSN: 2321-4007

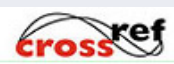

Identifiers and Pagination

Year: 2021 Vol: 14 No (6) Special Issue

Pages: 445- 448

This is an open access article under Creative
Commons License Attribn 4.0 Intl (CC-BY).
DOI: $h t t p: / / d x . d o i . o r g / 10.21786 / b b r c / 14.7 .93$

Commons License Attribn 4.0 Intl (CC-BY).
DOI: $h$ ttp://dx.doi.org/10.21786/bbrc/14.7.93 be sent to the doctor if any emergency regarding patient health.

A major advantage of the IoT is connectivity with available medical resources. One more concern with the current medical system is the manual, outdated, and error-prone system because the manual record-keeping in an enclosed file. This is cumbersome and also consumes a lot of energy and time that makes the medical response slow as well as tiresome but also terrible for both patients and clinic employees. The use of the cloud to store the health parameter as well as the patient history for people with the chronological disorder or any other issues can be easily managed and diagnosed by the doctor. Also, the admission of the patient to a hospital for a long time increases the financial burden on the family. Thus the use of IoT i.e. wearable sensors connectivity with any IoT enabled edge device that keeps monitoring the patient health at home as well as store day-wise medical history of the patient on the cloud. If anything goes beyond normalcy any trigging system to alert the doctor or the family member can be used.

2. Background: To use IoT service, edge devices are outfitted with sensors, actuators, processors, and transceivers. IoT is not a single entity but is a combination of various layered architecture which works together in tandem. Data collection

\section{Article Information}

ccepted after revision: $28^{\text {th }}$ July 2021
Received: $15^{\text {th }}$ May 2021 
from the sensors and process that data intelligently to retrieve the useful information from it. The data ingested by the sensors have to be stored and processed edge devices or different controller smartly to draw from useful results from it. Note that we generally define the term sensor; a cellular phone or even a some other electronics devices such as oven can term as a sensor as long as it gives inputs about its present state (internal value + environmental value).

An actuator is a kind ofhardware device that is used to derive a certain change in the environmental variable such as the temperature controller of an air conditioning system. The ingested data from the sensors will processed by controller or edge device. Further data can be sent to the remote server on the cloud system for future reference and to use that stored data remotely. Also due to financial limit to use cloud storage it is advisable to processed data on smart computing devices such as raspberry pi or other, and only the important or critical value will be sent to server on cloud that also reduces the financial burden. This is called fog computing where most of the analysis of the sensors data done on the edge devices. The storing capacity and processing ability of IoT devices are limited by the resources like energy consumption of the battery, size, and computational capability. Therefore the challenges to make any IoT solutions we need to get ensured about right data and accuracy.

Literature Review: In (P. Dinesh et. al.) it classifies technologicalchallenge that was required to work out the wide-scale agreement of smart and associated healthcare systems. It has explains the scope of IoT and big data technology that can hasten the pace at which e-health care system can be deployed, and embrace by all healthcare stakeholders. Digital healthcare systems that influence the use of internet of things and big data are expected to impeccably connect patients as well as health systems remotely across the globe. These systems are connected via internet to several medical wearable sensors technologies that are being used for real-time healthcare monitoring. A three-layer smart health care system had been proposed. In this paper, the author has an interface the medical sensor to get the health information, and also this system is equipped with the IoT gateway as well as with proper computing devices like computer used by the medical staff. When a medical checkup of a patient has done, data collected as of the wearable devices stored in the computing devices by using communication devices like Zigbee, Bluetooth, etc. smart health unit data has also stored on the local machine as well on the cloud, therefore, can be accessed from anywhere in the world.

The author has presented three layers; the sensor layer in which the sensor data has been collected with the help of the communication technologies like WiFi, ZigBee, LoWPAN, etc. The second layer is the network access layer which includes the IoT gateway which supports multiple communication technologies. The last layer was the service layer in which the collection has been stored on the remote server and can be retrieve anywhere in the world (Maruf Pasha et. al. (2018)).A system for remote health care is presented in which the author used the wireless technology Zigbee, which sends data to the raspberry pi2 $\mathrm{B}$ module which processes the data and sends data to the cloud. Amazon EC2 cloud model is used and then using the web page services health-related information can be addressed (Unnati Dhanaliya et. al. (2016)). The author presented an app-based ehealth care system in which individuals can register themselves after authenticating by hospital staff, they can receive their prescription and other medical-related information on the app. The author used the Google cloud messaging system for push notifications or any alert regarding medical emergencies (Md. Milon Islam et. al. (2020).

In this paper, a health care system using fog computing and cloud computing method is purposed. When data has been processed by the microcontroller, fog computing i.e. deep learning algorithm was used for pre-processing of information before sending that to the cloud ( $S$. Lakshmanachari et. al. (2017)). The author presented a machine learning framework for more accurate data processing of the patient health parameter using IoT. Arduino and sensor data are aggregated on Google sheet and then by collecting the data set of patient health, different types of machine learning classifiers are used to predict better efficiency (S. Lakshmanachari et. al. (2017)). Another paper in which mobile-based data acquisition system is purposed which collect the health-related data, store it processed it and can be further sent to the data center (Lakmini et. al. (2017)).

4. Proposed system: In this paper health monitoring system is proposed in which work is categorized into three different layers.

1. Sensor Integration layer 2. Fog layer 3. Cloud services

\section{Figure 1: Block diagram of e-health} care system using cloud

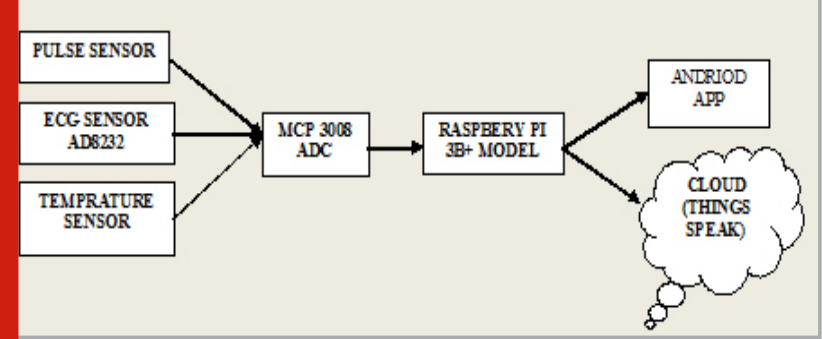

\section{Hardware Used:}

a) MCP3008 Analog to Digital Converter: As in raspberry pi have a limited option to interface the analog sensors directly. Thus to interface the analog sensors ECG and pulse sensors with pi, 10 bit analog to digital converter MCP3008 which having 8-channel is used. MCP3008 is a 16-pin IC out of which 8 pins are used to get analog input from $\mathrm{CH} 0-\mathrm{CH} 7$ (Pins 1-8) as shown in figure 2. Rest other pins are as follows:DGND-Digital ground Pin, CS-chip select pin, Din- data input from raspberry pi, Dout- output data pin, CLK-clock pin, AGND- analog ground pin, Vrefanalog reference voltage connected to 3.3 volt. VDD-power pin of IC. 
Figure 2: Interfacing of ADC MCP3008 to Raspberry Pi 3B+ (https://learn.adafruit.com/raspberry-pi-analog-todigital-converters/mcp3008, https://cdn-shop.adafruit.com/ datasheets/MCP3008.pdf)

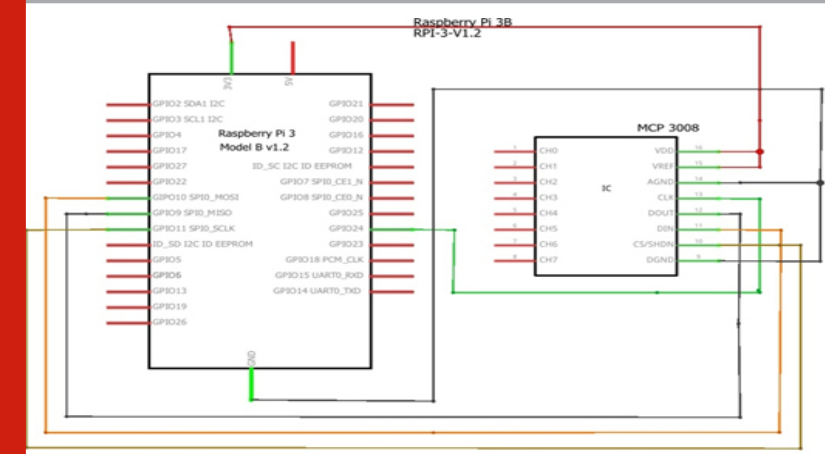

fritzing

b) Pulse Sensor:Pulse Sensor: Pulse Sensor is a 3 pin sensors having power, ground and signal pin. This sensor gives the heart rate when it is used on the finger tip or earlobe. Interfacing with MCP3008 ADC of pulse sensor is shown in figure 3 .

Figure 3 Interfacing of Pulse sensor with ADC (MCP3008) (https:/www.analog.com/.../technical-documentation/datasheets/AD8232.pdf)

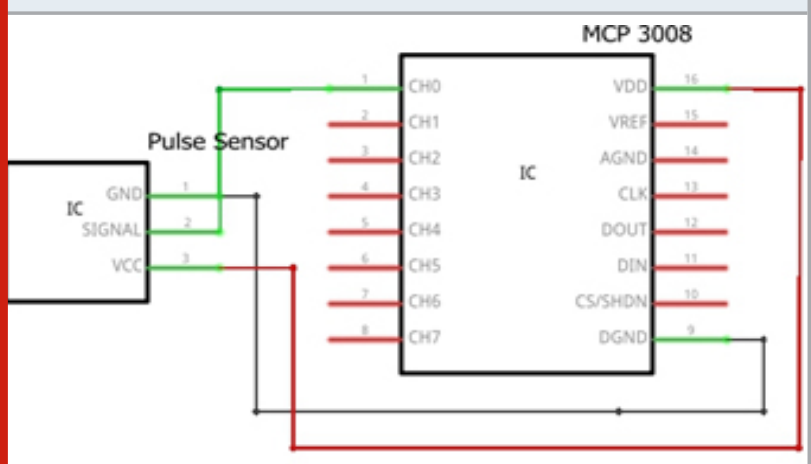

c) ECG Sensor: AD8232 analog IC ECG sensor is used in this project.Electrocardiography (ECG) monitors the electrical pulse of the heart over time. Variations over time, the magnitude of the electrical pulse, and morphology of the ECG signals are used for diagnosing anomalous heart related activity and conduction patterns. The AD8232 ECG sensor IC has nine pins for out of which GND, 3.3V, Output, $\mathrm{LO}+, \mathrm{LO}-$, SDNare important pins to operate the sensor with any development board. This IC has three more pins RL (Right Leg),RA (Right Arm) and LA (Left Arm) to attach the leads to body Also, there is an onboard LED indicator used for pulse rate. Therefore by using appropriately with a development board it fetches meaningful information from the collected data. Pin out and interfacing with MCP3008 ADC is shown in figure 4 (https://media.digikey.com/pdf/ Data Sheets/Pulse Sensor PDFs/Pulse_Sensor.pdf et. al.).

Design \& Analysis: Finally the proposed e-health care system has been implemented using the raspberry pi $3 \mathrm{~B}+$ model using the required medical sensors i.e. $\mathrm{ECG}$, pulse sensor and temperature as shown in figure 6 . After getting sensor integration done the data has been ingested to the thing speak cloud. Also an android based app also implemented that shows the real time data of the patient. Also a threshold for the pulse sensor and ECG sensor has been set if the value goes above the threshold limit a message also sent to the concerned medical staff or any family member.

Figure 4 (a) Interfacing of ECG sensor to ADC (MCP3008) (b) ECG sensor PIN diagram

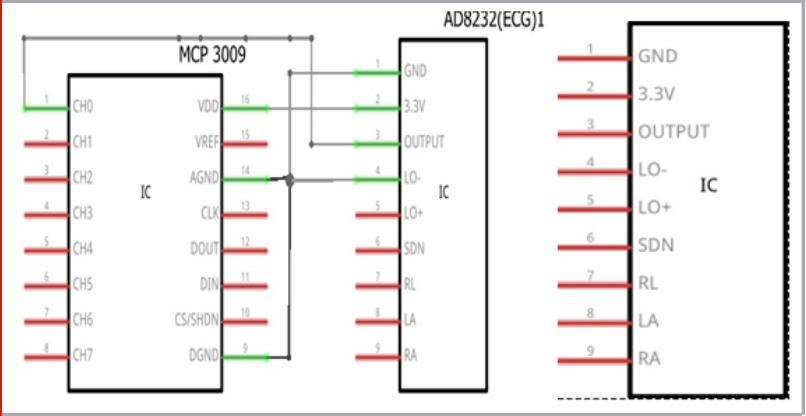

Table 1. Threshold limit for the Pulse Sensor and Temperature Sensor

\begin{tabular}{|l|c|}
\hline $\begin{array}{l}\text { Rhythm } \\
\text { type }\end{array}$ & $\begin{array}{c}\text { Threshold value of } \\
\text { Pulse Rate(PR) }\end{array}$ \\
\hline Normal Value & $\begin{array}{c}60 \leq \mathrm{PR} \leq 100 \\
\text { (heart beat /minute) }\end{array}$ \\
\hline $\begin{array}{l}\text { Condition for Bradcardia } \\
\text { (slower than normal) }\end{array}$ & $\mathrm{PR} \leq 60$ (heart beat /minute) \\
\hline $\begin{array}{l}\text { Condition for Tachycardia } \\
\text { (Higher than normal) }\end{array}$ & $\mathrm{PR} \geq 100$ (heart beat /minute) \\
\hline
\end{tabular}

Figure 5 (a): Data storage on Thingspeak cloud (b) Hardware configuration

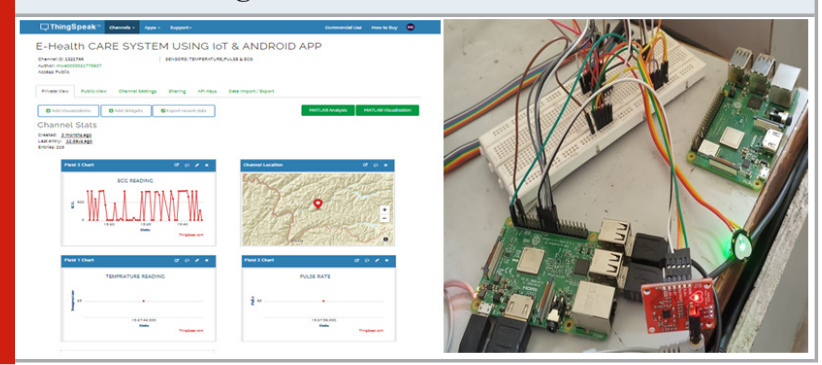

According to medical professional most of the people does not know that they are suffering from the high risk cardiac problem. So with the help of e-health solution this risk can be minimized if the sensors are properly calibrated with real time method or machine used in the medical terms. Therefore any sudden rise and fall in the pulse rate with in the early period of the patient before the cardiac arrest alarming message can be sent to the cardiologist so that in mean time necessary medical help can be provided those people. In such system a single physician can serve enough 
patients using this monitoring method along with his regular workload in the hospital.

\section{CONCLUSION \& FUTURE SCOPE}

In this paperwork we have developed remote health monitoring system which gives an insight that if a patient is suffering from a chronological disease, long illness or patients are residing in remote areas can diagnosed by doctor time to time by seeing the past history and present scenario. This system also helpful in emergency situation in which an alarm system is proposed so patient can get timely medical help or facility by the physician. As the computer technology evolves today and it is changing day by day we can improve the efficacy of such system so that the people who are living in the remote place or suffering from illness can get the medical facility timely. In future we can apply machine learning or deep learning algorithm to the patient medical history there is possibility to fetch any uncertainty to get more appropriate diagnosis by doctor.

\section{REFRENCES}

Andriy Romaniv, C.E.O., Gurjinder Singh, C.T.O., Yury Zykov, C.O.O., Hariri, N.B., Prithivi Kogulanathan, C.I.O. and Romaniv, A., 2020. PoC Prototype Design Specifications: Medeor.

Dhanaliya, U. and Devani, A., 2016, April. Implementation of e-health care system using web services and cloud computing. In 2016 International Conference on
Communication and Signal Processing (ICCSP) (pp. 1034-1036). IEEE.

Dineshkumar, P., SenthilKumar, R., Sujatha, K., Ponmagal, R.S. and Rajavarman, V.N., 2016, December. Big data analytics of IoT based Health care monitoring system. In 2016 IEEE Uttar Pradesh Section International Conference on Electrical, Computer and Electronics Engineering (UPCON) (pp. 55-60). IEEE.

Islam, M.M., Rahaman, A. and Islam, M.R., 2020. Development of smart healthcare monitoring system in IoT environment. SN computer science, 1, pp.1-11.

Lakshmanachari, S., Srihari, C., Sudhakar, A. and Nalajala, P., 2017, August. Design and implementation of cloud based patient health care monitoring systems using IoT. In 2017 International Conference on Energy, Communication, Data Analytics and Soft Computing (ICECDS) (pp. 37133717). IEEE.

Lakmini, P.M., Naeem, R. and Keshav, D., 2019. Remote patient monitoring: a comprehensive study: J. Ambient. Intell. Human. Comput.

Pasha, M. and Shah, S.M.W., 2018. Framework for E-Health systems in IoT-based environments. Wireless Communications and Mobile Computing, 2018.

Yang, C.N., Wu, F.H., Tsai, S.Y. and Kuo, W.C., 2015, December. E-health services for elderly care based on google cloud messaging. In 2015 IEEE International Conference on Smart City/SocialCom/SustainCom (SmartCity) (pp. 9-12). IEEE. 